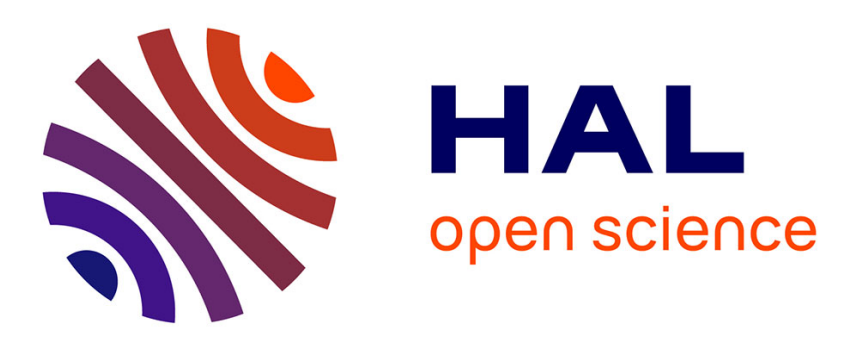

\title{
Hétérodiffusion du cérium dans le lanthane cubique centré. Effet de la transformation $\beta \rightarrow$ y sur les coefficients de diffusion
}

\author{
M. Fromont
}

\section{- To cite this version:}

M. Fromont. Hétérodiffusion du cérium dans le lanthane cubique centré. Effet de la transformation $\beta \rightarrow$ y sur les coefficients de diffusion. Journal de Physique Lettres, 1976, 37 (5), pp.117-118. 10.1051/jphyslet:01976003705011700 . jpa-00231251

HAL Id: jpa-00231251

https://hal.science/jpa-00231251

Submitted on 1 Jan 1976

HAL is a multi-disciplinary open access archive for the deposit and dissemination of scientific research documents, whether they are published or not. The documents may come from teaching and research institutions in France or abroad, or from public or private research centers.
L'archive ouverte pluridisciplinaire $\mathbf{H A L}$, est destinée au dépôt et à la diffusion de documents scientifiques de niveau recherche, publiés ou non, émanant des établissements d'enseignement et de recherche français ou étrangers, des laboratoires publics ou privés. 


\title{
HÉTÉRODIFFUSION DU CÉRIUM DANS LE LANTHANE CUBIQUE CENTRÉ EFFET DE LA TRANSFORMATION $\beta \rightarrow \gamma$ SUR LES COEFFICIENTS DE DIFFUSION
}

\author{
M. FROMONT \\ C.E.A., SEAMA Bt 19, C.E.N.F.A.R., B.P. no 6, 92260 Fontenay-aux-Roses, France
}

(Reçu le 13 février 1976, accepté le 4 mars 1976)

\begin{abstract}
Résumé. - Nous montrons que ni les dislocations créées au changement de phase : structure compacte-structure cubique centrée, ni les joints de grains ne peuvent être tenus pour responsables des coefficients de diffusion élevés dans les phases cubiques centrées des métaux de terres rares.
\end{abstract}

\begin{abstract}
It is shown that neither the dislocations resulting from the phase transformation (closed packed structure-body cubic centered structure), nor the grain boundaries are responsible for the high values of the diffusion coefficients in the b.c.c. structures of the rare earth metals.
\end{abstract}

Dans toutes les terres rares métalliques, qui possèdent la structure cubique centrée ont été trouvés pour cette phase des paramètres d'autodiffusion anormalement bas [1-5], exception faite pour l'europium [5]. L'europium étant précisément la seule terre rare à ne pas présenter de transformation solide-solide précédant la phase cubique centrée, il était utile de vérifier que cette transformation n'est pas à l'origine d'une création importante de dislocations, qui pourraient jouer ainsi le rôle de court-circuits de diffusion.

La similitude des paramètres de diffusion obtenus sur les couples $\mathrm{Ce}-141 / \mathrm{Ce}-\delta$ [2], La-140/Ce- $\delta$ [3] et La-140/La- $\gamma$ [3] laisse à penser que l'autodiffusion et l'hétérodiffusion dans le lanthane et le cérium sont régies par le même mécanisme de diffusion. Aussi avons-nous choisi d'étudier le couple Ce-141/La- $\gamma$.

$\mathrm{Si}$ les dislocations introduites par la transformation $\beta-\gamma$ du lanthane $\left(T_{\beta-\gamma}=865^{\circ} \mathrm{C}\right)$ accélèrent la diffusion, cet effet sera prépondérant en début de recuit, tant qu'elles n'auront pas été éliminées thermiquement. C'est pourquoi des recuits de différentes durées (dans le cas présent : $1 / 2$ heure, 1 heure et et 4 heures) effectués à la même température $\left(T=873^{\circ} \mathrm{C}\right)$ devraient conduire à des coefficients de diffusion plus élevés pour les recuits de courte durée dans l'hypothèse où les dislocations accéléreraient la diffusion. De même, en faisant traverser la transformation $\beta-\gamma$ toutes les 30 minutes à un échantillon $\mathrm{B}$ dont la température de palier est de $873^{\circ} \mathrm{C}$ durant 4 heures, on pouvait espérer montrer que l'effet des dislocations y est environ 8 fois plus important que dans le cas d'un échantillon A maintenu constamment à $873^{\circ} \mathrm{C}$ durant le même temps.

Les coefficients d'hétérodiffusion $D_{\mathrm{La}-\gamma}^{\mathrm{Ce}-141}$ ont été obtenus par la technique du dépôt électrolytique d'une couche mince de radiotraceur et la méthode d'abrasions et comptages.

En se reportant au tableau $I$, on peut voir que l'écart maximum entre les quatre coefficients de diffusion mesurés à $873{ }^{\circ} \mathrm{C}$ ne dépasse pas $5 \%$ ce qui est imputable aux erreurs expérimentales et non aux dislocations.

\section{TABLEAU I}

Hétérodiffusion du cérium 141 dans le lanthane $\gamma-\mathrm{CC}$

$\begin{array}{cccc}\begin{array}{c}\text { Température } \\ \text { en }{ }^{\circ} \mathrm{C}\end{array} & \begin{array}{c}\text { Durée du } \\ \text { recuit en s }\end{array} & \begin{array}{c}\text { Durée } \\ \text { corrigée }\end{array} & \begin{array}{c}\text { Coefficient de } \\ \text { diffusion } \mathrm{cm}^{2} \mathrm{~s}^{-1}\end{array} \\ 866 & 3180 & (3000 \pm 60) & (3,17 \pm 0,15) \times 10^{-7} \\ 873 & 1800 & 1601 & (3,23 \pm 0,08) \times 10^{-7} \\ 873 & 3600 & 3420 & (3,41 \pm 0,10) \times 10^{-7} \\ 873\left(^{*}\right) & 3600 & 3000 & (3,27 \pm 0,06) \times 10^{-7} \\ 873 & 14400 & 141642 & (3,25 \pm 0,07) \times 10^{-7} \\ 873\left(^{* *}\right) & 14400 & 130230 & (3,35 \pm 0,10) \times 10^{-7} \\ 883 & 3600 & 3474 & (3,80 \pm 0,13) \times 10^{-7} \\ 893 & 3600 & 3498 & (4,03 \pm 0,10) \times 10^{-7} \\ 897 & 4200 & 4102 & (4,11 \pm 0,08) \times 10^{-7}\end{array}$

(*) $10 \mathrm{mın}$. à $840^{\prime \prime} \mathrm{C}(\mathrm{La}-\beta)$ puis $50 \mathrm{~min}$. à $873^{\circ} \mathrm{C}$.

$(* *)$ Echantillon ayant subi 8 fois la transformation $\beta-\gamma$. 
Par ailleurs l'analyse du graphe d'Arrhénius conduit à une valeur du facteur de fréquence

$$
D_{0}=1,8 \times 10^{-2} \mathrm{~cm}^{2} \mathrm{~s}^{-1}
$$

et à une énergie d'activation $Q=25_{-3}^{+1,5} \mathrm{kcal} / \mathrm{mole}$, valeurs toutes deux caractéristiques d'un mode de diffusion anormale (la loi de Van Liempt $Q=34 T_{\mathrm{F}}$ où $T_{\mathrm{F}}$ est la température de fusion du métal étudié prévoit $Q=40,6 \mathrm{kcal} / \mathrm{mole}$ pour le lanthane $\gamma$ ). L'aspect bien linéaire des courbes log (activité spécifique $)=f\left(\right.$ pénétration $\left.^{2}\right)$ ainsi que les contrôles par autoradiographie permettent d'affirmer qu'il s'agit bien dans cette phase $\gamma$ du lanthane d'un mode de diffusion en volume (Fig. 1).

En conclusion, nous estimons que dans le lanthane, ainsi que dans toutes les autres terres rares cubiques centrées, ni les dislocations ni les joints de grains ne peuvent être tenus pour responsables des paramètres de diffusion trouvés anormalement bas au regard des lois couramment admises pour caractériser un mode de diffusion dit normal.

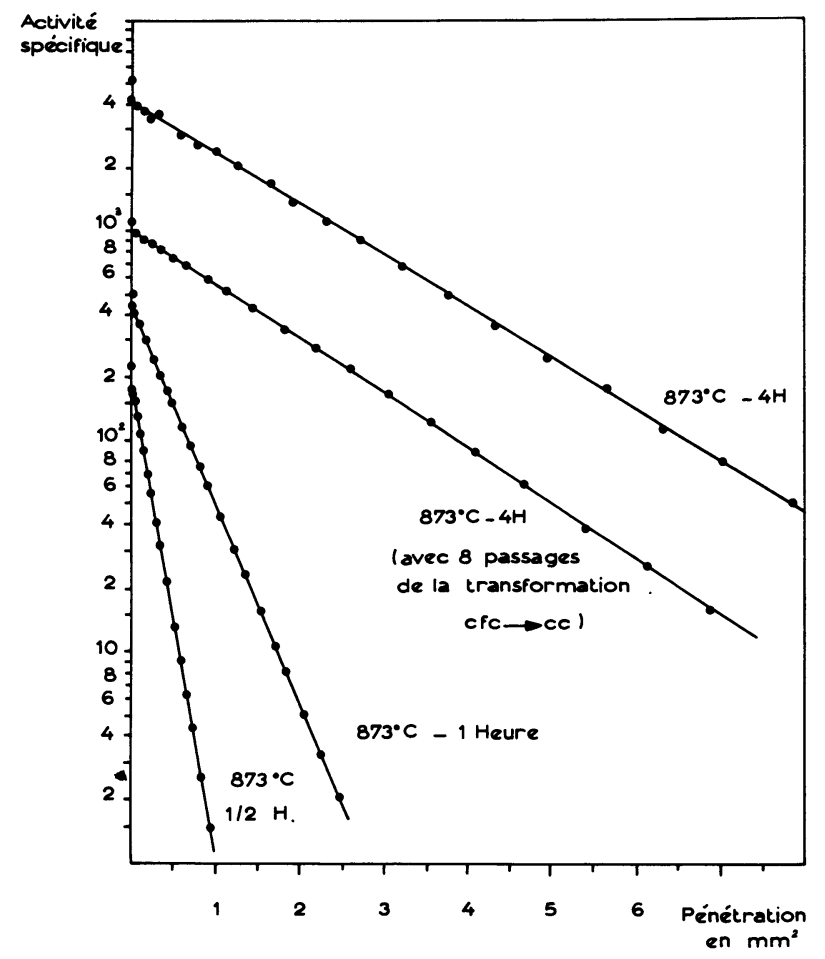

Fig. 1. - Exemples de courbes donnant la concentration en cérium en fonction du carré de la pénétration. Influence de la durée du recuit à température donnée : $873^{\circ} \mathrm{C}$.

\section{Bibliographie}

[1] Dariel, M. P., Erez, G., Schmidt, G. M. J., Phil. Mag. 19 (1969) 1045.

[2] Dariel, M. P., Dayan, D. et Languille, A., Phys. Rev. B, 4 (1971) 4348.
[3] Dariel, M. P., Phil. Mag. 28 (1973) 915.

[4] Fromont, M., Languille, A., Calais, D., J. Phys. \& Chem. Solids 35 (1974) 1367.

[5] Fromont, M., à paraître dans J. Phys. \& Chem. Solids. 